\title{
Time-Consistent Strategies for a Multiperiod Mean-Variance Portfolio Selection Problem
}

\author{
Huiling Wu \\ China Institute for Actuarial Science, Central University of Finance and Economics, Beijing 100081, China \\ Correspondence should be addressed to Huiling Wu; sunnyling168@hotmail.com
}

Received 1 January 2013; Accepted 28 February 2013

Academic Editor: Francis T. K. Au

Copyright (C) 2013 Huiling Wu. This is an open access article distributed under the Creative Commons Attribution License, which permits unrestricted use, distribution, and reproduction in any medium, provided the original work is properly cited.

It remained prevalent in the past years to obtain the precommitment strategies for Markowitz's mean-variance portfolio optimization problems, but not much is known about their time-consistent strategies. This paper takes a step to investigate the time-consistent Nash equilibrium strategies for a multiperiod mean-variance portfolio selection problem. Under the assumption that the risk aversion is, respectively, a constant and a function of current wealth level, we obtain the explicit expressions for the time-consistent Nash equilibrium strategy and the equilibrium value function. Many interesting properties of the time-consistent results are identified through numerical sensitivity analysis and by comparing them with the classical pre-commitment solutions.

\section{Introduction}

Since the pioneering work of [1] in a single period, meanvariance formulation has been one of focused topics of portfolio selection optimization and has stimulated hundreds of extensions and applications. The interested readers can refer to $[2,3]$ for detailed information. The objective of quite a number of existing mean-variance portfolio selection models is seeking an optimal strategy $\pi(\cdot)$ which maximizes the mean-variance utility $E\left[X_{T}^{\pi}\right]-\omega \operatorname{Var}\left[X_{T}^{\pi}\right]$, where $X_{T}^{\pi}$ is the terminal wealth. But it is well known that this mean-variance criterion lacks of iterated-expectation property, which gives rise to time-inconsistent investment strategy in the sense that Bellman optimality principle is not available any more. The so-called time-inconsistent strategy means that optimal strategy obtained at time $n$ does not agree with optimal strategy derived at time $m$ where $m>n$. Therefore, the optimal strategy in the classical time-inconsistent models is just optimal from the viewpoint of the initial time, and decision makers at any time $k$ after the initial time must commit themselves to the initial optimal strategy even if it is not optimal at time $k$. So, the time-inconsistent optimal strategy in the classical mean-variance model is called the precommitment strategy. But this precommitment has been criticized for lacking rationality. For one simple example, investment psychology and tastes will often change over time, and the decision maker at later time may not commit themselves to following a strategy which is not optimal at their current time. The work in [4] analyzed the incentives which induce the investor to revise her optimal strategy at subsequent dates under mean-variance criterion.

For this reason, we want to find an optimal strategy with time consistency which is necessary for a rational individual. The analysis of inconsistency can be traced back to [5] which pointed out that "optimal plan of the present moment is generally one which will not be obeyed" and the time-inconsistent problem can be solved by precommitment strategy or alternatively time-consistent strategy. The authors of $[5,6]$ devoted themselves to identifying an intertemporal consumption programme that would be "the best plan that an agent would actually follow." The work in [7] questioned the generality of the existence of StrotzPollak equilibrium and gave an alternative criterion of Nash equilibrium. More recently, it is of interest to study timeinconsistent problems. The work of $[8,9]$ investigated a time-consistent strategy for a consumption and investment problem with nonexponential discounting. The work in [10] gave general approaches to handle time-inconsistent 
problems by viewing them as a game theoretic framework and looking for Nash subgame perfect equilibrium points. They formally defined the continuous time equilibrium concept and derived the extension of $\mathrm{HJB}$ equation and its verification theorem for a very general objective functions. The work in [11] studied a continuous-time mean-variance portfolio optimization model on the assumption that the risk aversion factor depended dynamically on the current wealth. In view of the extension of HJB equation developed in [10], they obtained the time-consistent equilibrium control and equilibrium value function. The work in [12] provided explicit solutions to a series of cases, including mean-standard deviation in continuous-time setting. The work in [13] investigated optimal mean-variance time-consistent investment and reinsurance policies for an insurer under continuoustime setting. The work in [14] developed a fully numerical scheme to determine time-consistent mean-variance strategy based on piecewise constant policy technique. As for the discrete-time mean-variance models, the work in [15] gave a complicated backwards recursive relationship about timeconsistent investment strategy but had not found analytical expression for the strategy.

To the best of our knowledge, no existing literature has given time-consistent equilibrium strategy and equilibrium value function in closed form for discrete-time meanvariance asset allocation. Our research will fill the gap. We view this decision-making process as a noncooperative game and suppose that there is one decision maker, referred to as "decision maker $n$ ", for each point of time $n$. This assumption is reasonable. On one hand, in the real world, there are often quite different persons who will join in the decision-making process especially when the investment horizon is long; on the other hand, we can image decision-maker $n$ as the future incarnation of themselves at time $n$ considering that the tastes of the decision maker will change over time. So our work in this paper is listed as follows: (a) derive the analytical expressions for the time-consistent equilibrium strategy and equilibrium value function when the risk aversion is assumed to be a constant and a function of current wealth, respectively; (b) when risk aversion factor is a constant, compare our time-consistent results with the precommitment ones in [2] and present the particular properties of the time-consistent results; (c) study the problem in discrete-time setting with nonconstant risk aversion which is a function of current wealth and identify the properties of the investment proportion by numerical analysis.

The rest of the paper is organized as follows. In Section 2, the problem formulation is presented, and the recursive formula of the equilibrium value function is derived. In Section 3, equilibrium strategy and equilibrium value function for mean-variance model with constant risk aversion are obtained. Comparison of our time-consistent results with the precommitment ones in [2] is also given in this section. In Section 4, the equilibrium results are investigated on the assumption that the risk aversion depends dynamically on the current wealth and numerical analysis is given to demonstrate the properties of investment proportion. Section 5 presents our conclusions.

\section{Problem Formulation}

In this paper, we assume that investors join the market at time 0 with an initial wealth $x_{0}$ and plan to process the investment in $T$ consecutive time periods. There are one risk-free asset and $m_{1}$ risky assets in the market. The $m_{1}$ risky assets have random returns $R_{n}=\left(R_{n, 1}, R_{n, 2}, \ldots, R_{n, m_{1}}\right)^{\prime}$ at period $n$ (time interval $[n, n+1)$ ) where $R_{n, k}$ denotes the random return of the $k$ th asset at period $n$ and superscript " " stands for the transpose of a matrix or vector. Denote by $r_{n}^{f}$ the return of the risk-free asset at period $n$, and denote by $X_{n}$ and $\pi_{n}=\left(\pi_{n, 1}, \ldots, \pi_{n, m_{1}}\right)^{\prime}$ the wealth available for investment and the amounts invested in $m_{1}$ risky asset at time $n$, respectively. Then the wealth dynamics is

$$
X_{n+1}=\left(X_{n}-\sum_{k=1}^{m_{1}} \pi_{n, k}\right) r_{n}^{f}+\sum_{k=1}^{m_{1}} \pi_{n, k} R_{n, k}=X_{n} r_{n}^{f}+\pi_{n}^{\prime} R_{n}^{e},
$$

where $R_{n, k}^{e}=R_{n, k}-r_{n}^{f}, R_{n}^{e}=\left(R_{n, 1}^{e}, R_{n, 2}^{e}, \ldots, R_{n, m_{1}}^{e}\right)^{\prime}$ for $n=$ $0,1, \ldots, T-1$.

As we know, the classic mean-variance optimization problem is as follows:

$$
\max _{\left\{\pi_{0}, \pi_{1}, \ldots, \pi_{T-1}\right\}} E\left(X_{T}^{\pi}\right)-\omega \operatorname{Var}\left(X_{T}^{\pi}\right)
$$

which results in a time-inconsistent strategy, that is, precommitment strategy. Therefore, as mentioned in Section 1, this paper aims to solve this problem from another perspective and to look for the time-consistent Nash equilibrium investment strategy. To this end, we first give the definition of Nash equilibrium strategy according to [10] and the references therein.

Let $\pi(n)=\left\{\pi_{n}, \pi_{n+1}, \ldots, \pi_{T-1}\right\}$ be the policy made at time $n$ and

$$
J_{n}\left(\mathbb{F}_{n}, \pi(n)\right)=E\left(X_{T}^{\pi(n)} \mid \mathbb{F}_{n}\right)-\omega\left(\mathbb{F}_{n}\right) \operatorname{Var}\left(X_{T}^{\pi(n)} \mid \mathbb{F}_{n}\right),
$$

where $X_{T}^{\pi(n)}$ is the terminal wealth corresponding to the investment strategy $\pi$, and $\mathbb{F}_{n}$ is the information at time $n$, such as wealth level. A natural assumption is that risk aversion $\omega$ is a function of $\mathbb{F}_{n}$.

Definition 1. Let $\hat{\pi}$ be a fixed control law. For an arbitrary point $n(n=0,1, \ldots, T-1)$, one selects an arbitrary control value $\pi_{n}$ and define the strategy $\bar{\pi}(n)=\left(\pi_{n}, \widehat{\pi}_{n+1}, \ldots, \widehat{\pi}_{T-1}\right)$.

Then $\hat{\pi}$ is said to be a subgame perfect Nash equilibrium strategy (or simply equilibrium strategy) if for all $n<T$, it satisfies

$$
\max _{\pi_{n}} J_{n}\left(\mathbb{F}_{n} ; \bar{\pi}(n)\right)=J_{n}\left(\mathbb{F}_{n} ; \widehat{\pi}(n)\right) .
$$


In addition, if equilibrium strategy $\widehat{\pi}$ exists, the equilibrium value function is defined as

$$
V_{n}\left(\mathbb{F}_{n}\right)=J_{n}\left(\mathbb{F}_{n} ; \widehat{\pi}(n)\right)
$$

Let $\widehat{\pi}(n)$ be the Nash equilibrium strategy at time $n$, then Definition 1 makes it possible to solve the problem by the following procedure:

(a) $\hat{\pi}(T-1)=\widehat{\pi}_{T-1}=\arg \max _{\pi_{T-1}}\left[E\left(X_{T}^{\pi_{T-1}} \mid \mathbb{F}_{T-1}\right)-\right.$ $\left.\omega\left(\mathbb{F}_{T-1}\right) \operatorname{Var}\left(X_{T}^{\pi_{T-1}} \mid \mathbb{F}_{T-1}\right)\right]$;

(b) given that the decision maker $T-1$ will use $\hat{\pi}_{T-1}$, $\widehat{\pi}_{T-2}$ is the optimal control that optimizes objective function $J_{T-2}\left(\mathbb{F}_{T-2} ;\left(\pi_{T-2}, \widehat{\pi}_{T-1}\right)\right)$;

(c) generally, $\widehat{\pi}_{n}$ is obtained by letting decision maker $n$ choose $\pi_{n}$ to maximize $J_{n}$ given that the forthcoming decision makers $n+1, \ldots, T-1$ will choose the strategy $\hat{\pi}(n+1)=\left(\widehat{\pi}_{n+1}, \ldots, \widehat{\pi}_{T-1}\right)$; that is,

$$
\widehat{\pi}_{n}=\arg \max _{\pi_{n}} J_{n}\left(\mathbb{F}_{n} ;\left(\pi_{n}, \widehat{\pi}_{n+1}, \ldots, \widehat{\pi}_{T-1}\right)\right) .
$$

Now we try to derive the time-consistent Nash equilibrium strategy and value function, but first we need to give the following notations and assumptions throughout this paper:

(N1) $r_{n}^{e}=E\left(R_{n}^{e}\right), n=0,1, \ldots, T-1$;

(N2) $\sigma_{n}=E\left(R_{n}^{e} R_{n}^{e \prime}\right)-r_{n}^{e} r_{n}^{e \prime}, n=0,1, \ldots, T-1$;

(N3) $\kappa_{n}=r_{n}^{e^{\prime}}\left(\sigma_{n}\right)^{-1} r_{n}^{e}, n=0,1, \ldots, T-1$;

(N4) $g_{n}^{\pi(n)}\left(\mathbb{F}_{n}\right)=E\left[X_{T}^{\pi(n)} \mid \mathbb{F}_{n}\right], g_{n}\left(\mathbb{F}_{n}\right)=E\left[X_{T}^{\hat{\pi}(n)} \mid\right.$ $\left.\mathbb{F}_{n}\right], n=0,1, \ldots, T-1$;

(N5) $h_{n}^{\pi(n)}\left(\mathbb{F}_{n}\right)=E\left[\left(X_{T}^{\pi(n)}\right)^{2} \mid \mathbb{F}_{n}\right], h_{n}\left(\mathbb{F}_{n}\right)=E\left[\left(X_{T}^{\hat{\pi}(n)}\right)^{2} \mid\right.$ $\left.\mathbb{F}_{n}\right], n=0,1, \ldots, T-1$.

(A1) The distribution function of the random returns $R_{n}$ is $F_{n}$, and $\left\{R_{n}, n=0,1, \ldots, T-1\right\}$ is assumed to be statistically independent.

(A2) $\sigma_{n}$ is assumed to be positive definite.

(A3) Short selling is allowed for all risky assets in all periods. Unlimited borrowing and lending are permitted. Transaction costs are not taken into account.

(A4) Capital additions or withdrawals are forbidden for all assets in all periods.
With the notations above, we can obtain the recursions of $J_{n}$ and $V_{n}$. For the sake of convenience, we define $E_{n, \mathbb{F}_{n}}(\cdot)=$ $E\left[\cdot \mid \mathbb{F}_{n}\right]$ and $\operatorname{Var}_{n, \mathbb{F}_{n}}(\cdot)=\operatorname{Var}\left(\cdot \mid \mathbb{F}_{n}\right)$, then

$$
\begin{aligned}
& J_{n}\left(\mathbb{F}_{n} ; \pi(n)\right) \\
& =E_{n, \mathbb{F}_{n}}\left[J_{n+1}\left(\mathbb{F}_{n+1} ; \pi(n+1)\right)\right] \\
& -\left\{E _ { n , \mathbb { F } _ { n } } \left[E_{n+1, \mathbb{F}_{n+1}}\left(X_{T}^{\pi(n+1)}\right)\right.\right. \\
& \left.-\omega\left(\mathbb{F}_{n+1}\right) \operatorname{Var}_{n+1, \mathbb{F}_{n+1}}\left(X_{T}^{\pi(n+1)}\right)\right] \\
& \left.-E_{n, \mathbb{F}_{n}}\left(X_{T}^{\pi(n)}\right)+\omega\left(\mathbb{F}_{n}\right) \operatorname{Var}_{n, \mathbb{F}_{n}}\left(X_{T}^{\pi(n)}\right)\right\} \\
& =E_{n, \mathbb{F}_{n}}\left[J_{n+1}\left(\mathbb{F}_{n+1} ; \pi(n+1)\right)\right] \\
& -\left\{E_{n, \mathbb{F}_{n}}\left[E_{n+1, \mathbb{F}_{n+1}}\left(X_{T}^{\pi(n+1)}\right)\right]-E_{n, \mathbb{F}_{n}}\left(X_{T}^{\pi(n)}\right)\right\} \\
& +E_{n, \mathbb{F}_{n}}\left[\omega\left(\mathbb{F}_{n+1}\right) \operatorname{Var}_{n+1, \mathbb{F}_{n+1}}\left(X_{T}^{\pi(n+1)}\right)\right] \\
& -\omega\left(\mathbb{F}_{n}\right) \operatorname{Var}_{n, \mathbb{F}_{n}}\left(X_{T}^{\pi(n)}\right) \\
& =E_{n, \mathbb{F}_{n}}\left[J_{n+1}\left(\mathbb{F}_{n+1} ; \pi(n+1)\right)\right] \\
& +E_{n, \mathbb{F}_{n}}\left[\omega\left(\mathbb{F}_{n+1}\right) \operatorname{Var}_{n+1, \mathbb{F}_{n+1}}\left(X_{T}^{\pi(n+1)}\right)\right] \\
& -\omega\left(\mathbb{F}_{n}\right) \operatorname{Var}_{n, \mathbb{F}_{n}}\left(X_{T}^{\pi(n)}\right) \\
& =E_{n, \mathbb{F}_{n}}\left[J_{n+1}\left(\mathbb{F}_{n+1} ; \pi(n+1)\right)\right] \\
& +E_{n, \mathbb{F}_{n}}\left[\omega\left(\mathbb{F}_{n+1}\right) E_{n+1, \mathbb{F}_{n+1}}\left(X_{T}^{\pi(n+1)}\right)^{2}\right. \\
& \left.-\omega\left(\mathbb{F}_{n+1}\right)\left[E_{n+1, \mathbb{F}_{n+1}}\left(X_{T}^{\pi(n+1)}\right)\right]^{2}\right] \\
& -\omega\left(\mathbb{F}_{n}\right)\left[E_{n, \mathbb{F}_{n}}\left(X_{T}^{\pi(n)}\right)^{2}-\left[E_{n, \mathbb{F}_{n}}\left(X_{T}^{\pi(n)}\right)\right]^{2}\right] \\
& =E_{n, \mathbb{F}_{n}}\left[J_{n+1}\left(\mathbb{F}_{n+1} ; \pi(n+1)\right)\right] \\
& +E_{n, \mathbb{F}_{n}}\left[\omega\left(\mathbb{F}_{n+1}\right) E_{n+1, \mathbb{F}_{n+1}}\left(\left(X_{T}^{\pi(n+1)}\right)^{2}\right)\right] \\
& -\omega\left(\mathbb{F}_{n}\right) E_{n, \mathbb{F}_{n}}\left(\left(X_{T}^{\pi(n)}\right)^{2}\right) \\
& -E_{n, \mathbb{F}_{n}}\left[\omega\left(\mathbb{F}_{n+1}\right)\left[E_{n+1, \mathbb{F}_{n+1}}\left(X_{T}^{\pi(n+1)}\right)\right]^{2}\right] \\
& +\omega\left(\mathbb{F}_{n}\right)\left\{E_{n, \mathbb{F}_{n}}\left[E_{n+1, \mathbb{F}_{n+1}}\left(X_{T}^{\pi(n+1)}\right)\right]\right\}^{2} \\
& =E_{n, \mathbb{F}_{n}}\left[J_{n+1}\left(\mathbb{F}_{n+1} ; \pi(n+1)\right)\right] \\
& +E_{n, \mathbb{F}_{n}}\left[\omega\left(\mathbb{F}_{n+1}\right) h_{n+1}^{\pi(n+1)}\left(\mathbb{F}_{n+1}\right)\right] \\
& -\omega\left(\mathbb{F}_{n}\right) E_{n, \mathbb{F}_{n}}\left(h_{n+1}^{\pi(n+1)}\left(\mathbb{F}_{n+1}\right)\right) \\
& -E_{n, \mathbb{F}_{n}}\left[\omega\left(\mathbb{F}_{n+1}\right)\left[g_{n+1}^{\pi(n+1)}\left(\mathbb{F}_{n+1}\right)\right]^{2}\right] \\
& +\omega\left(\mathbb{F}_{n}\right)\left[E_{n, \mathbb{F}_{n}}\left(g_{n+1}^{\pi(n+1)}\left(\mathbb{F}_{n+1}\right)\right)\right]^{2} .
\end{aligned}
$$


By Definition 1, we have $V_{n+1}\left(\mathbb{F}_{n+1}\right)=J_{n+1}\left(\mathbb{F}_{n+1} ; \widehat{\pi}_{n+1}\right)$, and hence (7) implies the following recursion for equilibrium value function $V_{n}\left(\mathbb{F}_{n}\right)$ :

$$
\begin{aligned}
& V_{n}\left(\mathbb{F}_{n}\right)=\max _{\pi_{n}}\{ E_{n, \mathbb{F}_{n}}\left[V_{n+1}\left(\mathbb{F}_{n+1}\right)\right] \\
&+E_{n, \mathbb{F}_{n}}\left[\omega\left(\mathbb{F}_{n+1}\right) h_{n+1}\left(\mathbb{F}_{n+1}\right)\right] \\
&-\omega\left(\mathbb{F}_{n}\right) E_{n, \mathbb{F}_{n}}\left(h_{n+1}\left(\mathbb{F}_{n+1}\right)\right) \\
&-E_{n, \mathbb{F}_{n}}\left[\omega\left(\mathbb{F}_{n+1}\right)\left[g_{n+1}\left(\mathbb{F}_{n+1}\right)\right]^{2}\right] \\
&\left.+\omega\left(\mathbb{F}_{n}\right)\left[E_{n, \mathbb{F}_{n}}\left(g_{n+1}\left(\mathbb{F}_{n+1}\right)\right)\right]^{2}\right\}, \\
& n=0,1, \ldots, T-1, \\
& V_{T}\left(\mathbb{F}_{n}\right)=x,
\end{aligned}
$$

where the recursions of $g_{n}\left(\mathbb{F}_{n}\right)$ and $h_{n}\left(\mathbb{F}_{n}\right)$ are as follows:

$$
\begin{gathered}
h_{n}\left(\mathbb{F}_{n}\right)=E_{n, \mathbb{F}_{n}}\left[\left(X_{T}^{\widehat{\pi}(n)}\right)^{2}\right] \\
=E_{n, \mathbb{F}_{n}}\left[E_{n+1, \mathbb{F}_{n+1}}\left(\left(X_{T}^{\widehat{\pi}(n)}\right)^{2}\right)\right] \\
=E_{n, \mathbb{F}_{n}}\left(h_{n+1}\left(\mathbb{F}_{n+1}\right)\right), \quad n=0,1, \ldots, T-1, \\
h_{T}\left(\mathbb{F}_{T}\right)=x^{2}, \\
g_{n}\left(\mathbb{F}_{n}\right)=E_{n, \mathbb{F}_{n}}\left(X_{T}^{\widehat{\pi}(n)}\right) \\
=E_{n, \mathbb{F}_{n}}\left[E_{n+1, \mathbb{F}_{n+1}}\left(X_{T}^{\widehat{\pi}(n)}\right)\right]=E_{n, \mathbb{F}_{n}}\left(g_{n+1}\left(\mathbb{F}_{n+1}\right)\right), \\
g_{T}\left(\mathbb{F}_{T}\right)=x .
\end{gathered}
$$

\section{Nash Equilibrium Strategy with Constant Risk Aversion}

3.1. Equilibrium Strategy and Equilibrium Value Function. When the risk aversion is a constant, $J_{n}$ is of the form

$$
\begin{gathered}
J_{n}(x ; \pi(n))=E\left(X_{T}^{\pi(n)} \mid X_{n}=x\right)-\omega \operatorname{Var}\left(X_{T}^{\pi(n)} \mid X_{n}=x\right) \\
V_{n}\left(\mathbb{F}_{n}\right)=V_{n}\left(X_{n}=x\right)=J_{n}(x ; \widehat{\pi}(n)) .
\end{gathered}
$$

According to (8), the recursion of equilibrium value function $V_{n}(x)$ is simplified as

$$
\begin{gathered}
V_{n}(x)=\max _{\pi_{n}}\left\{E_{n, x}\left[V_{n+1}\left(X_{n+1}^{\pi_{n}}\right)\right]\right. \\
\left.-\omega \operatorname{Var}_{n, x}\left[g_{n+1}\left(X_{n+1}^{\pi_{n}}\right)\right]\right\}, \\
n=0,1, \ldots, T-1, \\
V_{T}(x)=x .
\end{gathered}
$$

Recursion (12) indicates that $V_{n}(x)$ does not depend on $h_{n}$, and then we only need to find the explicit expression of $g_{n}$ by the following recursion:

$$
\begin{aligned}
g_{n}(x) & =E_{n, x}\left(X_{T}^{\widehat{\pi}(n)}\right) \\
& =E_{n, x}\left[g_{n+1}\left(X_{n+1}^{\widehat{\pi}_{n}}\right)\right], \quad n=0,1, \ldots, T-1, \\
g_{T}(x) & =x .
\end{aligned}
$$

The following theorem gives the explicit expressions of $\hat{\pi}$ and $V_{n}(x)$

Theorem 2. When the risk aversion is a constant, the Nash equilibrium strategy is given by

$$
\widehat{\pi}_{n}=\frac{1}{2 \omega} \frac{1}{\prod_{k=n+1}^{T-1} r_{k}^{f}}\left(\sigma_{n}\right)^{-1} r_{n}^{e}, \quad n=0,1, \ldots, T-1 .
$$

The corresponding equilibrium value function is

$$
\begin{aligned}
& V_{n}(x)=x \prod_{k=n}^{T-1} r_{k}^{f}+\frac{1}{4 \omega} \sum_{k=n}^{T-1} r_{k}^{e^{\prime}}\left(\sigma_{k}\right)^{-1} r_{k}^{e}, \quad n=0,1, \ldots, T, \\
& g_{n}(x)=x \prod_{k=n}^{T-1} r_{k}^{f}+\frac{1}{2 \omega} \sum_{k=n}^{T-1} r_{k}^{e^{\prime}}\left(\sigma_{k}\right)^{-1} r_{k}^{e}, \quad n=0,1, \ldots, T .
\end{aligned}
$$

Proof. Obviously (17) and (18) hold true for $n=T$. Then for $n=T-1$,

$$
\begin{aligned}
V_{T-1}(x)=\max _{\pi_{T-1}}\left\{E_{T-1, x}\left[V_{T}\left(X_{T}^{\pi_{T-1}}\right)\right]\right. \\
\left.\quad-\omega \operatorname{Var}_{T-1, x}\left[g_{T}\left(X_{T}^{\pi_{T-1}}\right)\right]\right\} \\
=\max _{\pi_{T-1}}\left[E_{T-1, x}\left(X_{T}^{\pi_{T-1}}\right)-\omega \operatorname{Var}_{T-1, x}\left(X_{T}^{\pi_{T-1}}\right)\right] \\
=x r_{T-1}^{f}+\sup _{\pi_{T-1}}\left[\pi_{T-1}^{\prime} r_{T-1}^{e}-\omega \pi_{T-1}^{\prime} \sigma_{T-1} \pi_{T-1}\right] .
\end{aligned}
$$

Since $\sigma_{T-1}$ is positive definite, the optimal solution exists and is given by

$$
\widehat{\pi}_{T-1}=\frac{1}{2 \omega}\left(\sigma_{T-1}\right)^{-1} r_{T-1}^{e} .
$$

Substituting (20) into (19) yields

$$
\begin{gathered}
V_{T-1}(x)=x r_{T-1}^{f}+\frac{1}{4 \omega} r_{T-1}^{e}{ }^{\prime} \sigma_{T-1}^{-1} r_{T-1}^{e}, \\
g_{T-1}(x)=E_{T-1, x}\left[X_{T}^{\hat{\pi}_{T-1}}\right]=x r_{T-1}^{f}+\frac{1}{2 \omega} r_{T-1}^{e}{ }^{\prime} \sigma_{T-1}^{-1} r_{T-1}^{e} .
\end{gathered}
$$


Hence (16), (17), and (18) hold true for $n=T-1$. Now we assume that (17) and (18) are true for $n+1$, then for $n$,

$$
\begin{aligned}
& V_{n}(x)=\max _{\pi_{n}}\left\{E_{n, x}\left[V_{n+1}\left(X_{n+1}^{\pi_{n}}\right)\right]\right. \\
& \left.-\omega \operatorname{Var}_{n, x}\left[g_{n+1}\left(X_{n+1}^{\pi_{n}}\right)\right]\right\} \\
& =\max _{\pi_{n}}\left\{E _ { n , x } \left[\left(x r_{n}^{f}+R_{n}^{e \prime} \pi_{n}\right) \prod_{k=n+1}^{T-1} r_{k}^{f}\right.\right. \\
& \left.+\frac{1}{4 \omega} \sum_{k=n+1}^{T-1} r_{k}^{e \prime}\left(\sigma_{k}\right)^{-1} r_{k}^{e}\right] \\
& -\omega \operatorname{Var}_{n, x}\left[\left(x r_{n}^{f}+R_{n}^{e \prime} \pi_{n}\right) \prod_{k=n+1}^{T-1} r_{k}^{f}\right. \\
& \left.\left.+\frac{1}{2 \omega} \sum_{k=n+1}^{T-1} r_{k}^{e^{\prime}}\left(\sigma_{k}\right)^{-1} r_{k}^{e}\right]\right\} \\
& =\max _{\pi_{n}}\left\{x \prod_{k=n}^{T-1} r_{k}^{f}+r_{n}^{e \prime} \pi_{n} \prod_{k=n+1}^{T-1} r_{k}^{f}\right. \\
& +\frac{1}{4 \omega} \sum_{k=n+1}^{T-1} r_{k}^{e \prime}\left(\sigma_{k}\right)^{-1} r_{k}^{e} \\
& \left.-\omega \prod_{k=n+1}^{T-1}\left(r_{k}^{f}\right)^{2} \pi_{n}^{\prime} \sigma_{n} \pi_{n}\right\} .
\end{aligned}
$$

It is obvious that the optimal solution of (22) exits and is given by

$$
\widehat{\pi}_{n}=\frac{1}{2 \omega} \frac{1}{\prod_{k=n+1}^{T-1} r_{k}^{f}}\left(\sigma_{n}\right)^{-1} r_{n}^{e} .
$$

Substituting (23) into (22), we obtain

$$
V_{n}(x)=x \prod_{k=n}^{T-1} r_{k}^{f}+\frac{1}{4 \omega} \sum_{k=n}^{T-1} r_{k}^{e \prime}\left(\sigma_{k}\right)^{-1} r_{k}^{e},
$$

and according to (14),

$$
\begin{aligned}
g_{n}(x)=E_{n, x} & {\left[g_{n+1}\left(X_{n+1}^{\widehat{\pi}_{n}}\right)\right] } \\
=E_{n, x} & {\left[X_{n+1}^{\widehat{\pi}_{n}} \prod_{k=n+1}^{T-1} r_{k}^{f}\right.} \\
& \left.+\frac{1}{2 \omega} \sum_{k=n+1}^{T-1} r_{k}^{e^{\prime}}\left(\sigma_{k}\right)^{-1} r_{k}^{e}\right]
\end{aligned}
$$

$$
\begin{aligned}
= & \left(x r_{n}^{f}+\widehat{\pi}_{n} r_{n}^{e}\right) \prod_{k=n+1}^{T-1} r_{k}^{f} \\
& +\frac{1}{2 \omega} \sum_{k=n+1}^{T-1} r_{k}^{e^{\prime}}\left(\sigma_{k}\right)^{-1} r_{k}^{e} \\
= & x \prod_{k=n}^{T-1} r_{k}^{f}+\frac{1}{2 \omega} r_{n}^{e^{\prime}}\left(\sigma_{n}\right)^{-1} r_{n}^{e} \\
& +\frac{1}{2 \omega} \sum_{k=n+1}^{T-1} r_{k}^{e^{\prime}}\left(\sigma_{k}\right)^{-1} r_{k}^{e} \\
= & x \prod_{k=n}^{T-1} r_{k}^{f}+\frac{1}{2 \omega} \sum_{k=n}^{T-1} r_{k}^{e^{\prime}}\left(\sigma_{k}\right)^{-1} r_{k}^{e} .
\end{aligned}
$$

Equations (23), (24), and (25) mean that (16), (17), and (18) hold true for $n$. By induction, the proof of Theorem 2 is complete.

\subsection{Comparison to the Precommitment Results}

3.2.1. About the Value Function. In view of (17), we know that the equilibrium value function at initial time 0 is

$$
V_{0}\left(x_{0}\right)=x_{0} \prod_{k=0}^{T-1} r_{k}^{f}+\frac{1}{4 \omega} \sum_{k=0}^{T-1} r_{k}^{e^{\prime}}\left(\sigma_{k}\right)^{-1} r_{k}^{e},
$$

and the precommitment value function of [2] is

$$
\begin{aligned}
E_{0, x_{0}}\left(X_{T}\right)-\omega \operatorname{Var}_{0, x_{0}}\left(X_{T}\right) & \\
= & x_{0} \prod_{k=0}^{T-1} r_{k}^{f} \\
& +\frac{1}{4 \omega} \frac{1-\prod_{k=0}^{T-1}\left(1-r_{k}^{e \prime} E^{-1}\left(R_{k}^{e} R_{k}^{e \prime}\right) r_{k}^{e}\right)}{\prod_{k=0}^{T-1}\left(1-r_{k}^{e \prime} E^{-1}\left(R_{k}^{e} R_{k}^{e \prime}\right) r_{k}^{e}\right)} .
\end{aligned}
$$

The relationship between (26) and (27) is summarized in the following lemma.

Lemma 3. Consider the following:

$$
E_{0, x_{0}}\left(X_{T}\right)-\omega \operatorname{Var}_{0, x_{0}}\left(X_{T}\right) \geq V_{0}\left(x_{0}\right) .
$$

Proof. First of all, we have

$$
E^{-1}\left(R_{k}^{e} R_{k}^{e^{\prime}}\right)=\left(\sigma_{k}\right)^{-1}-\frac{\left(\sigma_{k}\right)^{-1} r_{k}^{e} r_{k}^{e \prime}\left(\sigma_{k}\right)^{-1}}{1+r_{k}^{e \prime}\left(\sigma_{k}\right)^{-1} r_{k}^{e}},
$$


then,

$$
\begin{aligned}
r_{k}^{e \prime} E^{-1} & \left(R_{k}^{e} R_{k}^{e^{\prime}}\right) r_{k}^{e} \\
& =r_{k}^{e \prime}\left[\left(\sigma_{k}\right)^{-1}-\frac{\left(\sigma_{k}\right)^{-1} r_{k}^{e} r_{k}^{e^{\prime}}\left(\sigma_{k}\right)^{-1}}{1+r_{k}^{e^{\prime}}\left(\sigma_{k}\right)^{-1} r_{k}^{e}}\right] r_{k}^{e} \\
& =r_{k}^{e^{\prime}}\left(\sigma_{k}\right)^{-1} r_{k}^{e}\left[1-\frac{r_{k}^{e^{\prime}}\left(\sigma_{k}\right)^{-1} r_{k}^{e}}{1+r_{k}^{e^{\prime}}\left(\sigma_{k}\right)^{-1} r_{k}^{e}}\right] \\
& =\frac{r_{k}^{e^{\prime}}\left(\sigma_{k}\right)^{-1} r_{k}^{e}}{1+r_{k}^{e^{\prime}}\left(\sigma_{k}\right)^{-1} r_{k}^{e}} .
\end{aligned}
$$

Now,

$$
\begin{aligned}
& E_{0, x_{0}}\left(X_{T}\right)-\omega \operatorname{Var}_{0, x_{0}}\left(X_{T}\right)-V_{0}\left(x_{0}\right) \\
& =\frac{1}{4 \omega}\left[\frac{1-\prod_{k=0}^{T-1}\left(1-r_{k}^{e \prime} E^{-1}\left(R_{k}^{e} R_{k}^{e^{\prime}}\right) r_{k}^{e}\right)}{\prod_{k=0}^{T-1}\left(1-r_{k}^{e^{\prime}} E^{-1}\left(R_{k}^{e} R_{k}^{e^{\prime}}\right) r_{k}^{e}\right)}\right. \\
& \left.-\sum_{k=0}^{T-1} r_{k}^{e^{\prime}}\left(\sigma_{k}\right)^{-1} r_{k}^{e}\right] \\
& =\frac{1}{4 \omega} \frac{1-\prod_{k=0}^{T-1}\left(1 /\left(1+r_{k}^{e^{\prime}}\left(\sigma_{k}\right)^{-1} r_{k}^{e}\right)\right)}{\prod_{k=0}^{T-1}\left(1 /\left(1+r_{k}^{e}\left(\sigma_{k}\right)^{-1} r_{k}^{e}\right)\right)} \\
& -\frac{1}{4 \omega} \sum_{k=0}^{T-1} r_{k}^{e^{\prime}}\left(\sigma_{k}\right)^{-1} r_{k}^{e} \\
& =\frac{1}{4 \omega} \prod_{k=0}^{T-1}\left(1+r_{k}^{e \prime}\left(\sigma_{k}\right)^{-1} r_{k}^{e}\right) \\
& -\frac{1}{4 \omega} \sum_{k=0}^{T-1} r_{k}^{e \prime}\left(\sigma_{k}\right)^{-1} r_{k}^{e}-\frac{1}{4 \omega} \\
& =\frac{1}{4 \omega}\left[\left(1+r_{0}^{e \prime}\left(\sigma_{0}\right)^{-1} r_{0}^{e}\right) \cdots\left(1+r_{T-1}^{e}{ }^{\prime}\left(\sigma_{T-1}\right)^{-1} r_{T-1}^{e}\right)\right. \\
& \left.-\sum_{k=0}^{T-1} r_{k}^{e^{\prime}}\left(\sigma_{k}\right)^{-1} r_{k}^{e}-1\right] \geq 0
\end{aligned}
$$

This proof gives an important inequality needed in the later analysis as

$$
\begin{gathered}
\frac{1-\prod_{k=0}^{T-1}\left(1-r_{k}^{e \prime} E^{-1}\left(R_{k}^{e} R_{k}^{e \prime}\right) r_{k}^{e}\right)}{\prod_{k=0}^{T-1}\left(1-r_{k}^{e \prime} E^{-1}\left(R_{k}^{e} R_{k}^{e \prime}\right) r_{k}^{e}\right)}-\sum_{k=0}^{T-1} r_{k}^{e \prime}\left(\sigma_{k}\right)^{-1} r_{k}^{e} \\
=\left(1+r_{0}^{e^{\prime}}\left(\sigma_{0}\right)^{-1} r_{0}^{e}\right) \cdots\left(1+r_{T-1}^{e}{ }^{\prime}\left(\sigma_{T-1}\right)^{-1} r_{T-1}^{e}\right) \\
\quad-\sum_{k=0}^{T-1} r_{k}^{e^{\prime}}\left(\sigma_{k}\right)^{-1} r_{k}^{e}-1 \geq 0
\end{gathered}
$$

Lemma 3 shows that the precommitment value function is greater than the equilibrium value function. This is a fair game of God. The Nash equilibrium strategy gains the time consistency but at the same time destroys the welfare of the whole decision procedure because of the inability to precommit. Referring to the proof of Lemma 3, we also realize that the distance between these two value functions is amplified at longer time horizon. Specially, when $T=1$, these two value functions coincide with each other. When $T=1$, the time-consistent results should be and are actually the same as the precommitment ones.

3.2.2. About the Investment Strategy. The time-consistent strategy at each period is

$$
\widehat{\pi}_{n}=\frac{1}{2 \omega} \frac{1}{\prod_{k=n+1}^{T-1} r_{k}^{f}}\left(\sigma_{n}\right)^{-1} r_{n}^{e}, \quad n=0,1, \ldots, T-1
$$

Referring to [2], the precommitment strategy at each period is

$$
\begin{aligned}
\pi_{n}^{*}= & -r_{n}^{f} E^{-1}\left(R_{n}^{e} R_{n}^{e^{\prime}}\right) r_{n}^{e} x_{n} \\
& +\left[\prod_{k=0}^{T-1} r_{k}^{f} x_{0}+\frac{1}{2 \omega\left(\prod_{k=0}^{T-1}\left(1-r_{k}^{e} E^{-1}\left(R_{k}^{e} R_{k}^{e}\right) r_{k}^{e}\right)\right)}\right] \\
& \times \prod_{k=n+1}^{T-1}\left(\frac{1}{r_{k}^{f}}\right) E^{-1}\left(R_{n}^{e} R_{n}^{e^{\prime}}\right) r_{n}^{e}, \\
& \quad n=0,1, \ldots, T-1 .
\end{aligned}
$$

The significant differences between $\hat{\pi}$ and $\pi^{*}$ are as follows.

(a) Since the time-consistent strategy at time $n$ will not be affected by the initial information, then it has nothing to do with the initial wealth $x_{0}$ in contrast with precommitment strategy.

(b) the time consistent is time deterministic but the precommitment one is stochastically dependent on the current wealth.

3.2.3. About the Efficient Frontier. In this Section, we want to compare our efficient frontier with the one in [2]. But first of all, we need the results in Lemma 4. 
Lemma 4. Under the time-consistent strategy (16),

$$
\begin{aligned}
E\left(X_{n}^{\widehat{\pi}}\right) & =x_{0} \prod_{k=0}^{n-1} r_{k}^{f}+\frac{1}{2 \omega} \frac{1}{\prod_{k=n}^{T-1} r_{k}^{f}} \sum_{k=0}^{n-1} r_{k}^{e^{\prime}}\left(\sigma_{k}\right)^{-1} r_{k}^{e}, \\
E\left[\left(X_{n}^{\widehat{\pi}}\right)^{2}\right] & \\
= & x_{0} \prod_{k=0}^{n-1}\left(r_{k}^{f}\right)^{2}+\frac{1}{\omega} \frac{\sum_{k=0}^{n-1} r_{k}^{e^{\prime}}\left(\sigma_{k}\right)^{-1} r_{k}^{e}}{\prod_{k=n}^{T-1}\left(r_{k}^{f}\right)^{2}} x_{0} \prod_{k=0}^{T-1} r_{k}^{f} \\
& +\frac{1}{4 \omega^{2}} \frac{1}{\prod_{k=n}^{T-1}\left(r_{k}^{f}\right)^{2}} \sum_{k=0}^{n-1} r_{k}^{e \prime}\left(\sigma_{k}\right)^{-1} r_{k}^{e} \\
& \times\left[1+\sum_{m=0}^{k-1} r_{m}^{e}{ }^{\prime}\left(\sigma_{m}\right)^{-1} r_{m}^{e}+\sum_{m=0}^{k} r_{m}^{e}\left(\sigma_{m}\right)^{-1} r_{m}^{e}\right]
\end{aligned}
$$

Proof. Substituting (16) into (1) yields

$$
\begin{aligned}
X_{n}^{\widehat{\pi}}= & X_{n-1}^{\hat{\pi}} r_{n-1}^{f}+\frac{1}{2 \omega} \frac{1}{\prod_{k=n}^{T-1} r_{k}^{f}} \\
& \times R_{n-1}^{e}{ }^{\prime}\left(\sigma_{n-1}\right)^{-1} r_{n-1}^{e}, \quad n=1,2, \ldots, T,
\end{aligned}
$$

and for $n=1,2, \ldots, T$,

$$
\begin{aligned}
\left(X_{n}^{\widehat{\pi}}\right)^{2}= & \left(X_{n-1}^{\widehat{\pi}}\right)^{2}\left(r_{n-1}^{f}\right)^{2} \\
& +\frac{1}{\omega} X_{n-1}^{\widehat{\pi}} r_{n-1}^{f} \frac{1}{\prod_{k=n}^{T-1} r_{k}^{f}} R_{n-1}^{e}{ }^{\prime}\left(\sigma_{n-1}\right)^{-1} r_{n-1}^{e} \\
& +\frac{1}{4 \omega^{2}} \frac{1}{\prod_{k=n}^{T-1}\left(r_{k}^{f}\right)^{2}} r_{n-1}^{e}{ }^{\prime}\left(\sigma_{n-1}\right)^{-1} \\
& \times R_{n-1}^{e} R_{n-1}^{e}{ }^{\prime}\left(\sigma_{n-1}\right)^{-1} r_{n-1}^{e} .
\end{aligned}
$$

Hence,

$$
\begin{aligned}
E\left(X_{n}^{\widehat{\pi}}\right)= & E\left(X_{n-1}^{\widehat{\hat{n}}}\right) r_{n-1}^{f} \\
& +\frac{1}{2 \omega} \frac{1}{\prod_{k=n}^{T-1} r_{k}^{f}} r_{n-1}^{e}{ }^{\prime}\left(\sigma_{n-1}\right)^{-1} r_{n-1}^{e}, \\
& \quad n=1,2, \ldots, T,
\end{aligned}
$$

and for $n=1,2, \ldots, T$,

$$
\begin{aligned}
E\left[\left(X_{n}^{\widehat{\pi}}\right)^{2}\right] & \\
= & E\left(X_{n-1}^{\widehat{\pi}}\right)^{2}\left(r_{n-1}^{f}\right)^{2}+\frac{1}{\omega} E\left(X_{n-1}^{\widehat{\pi}}\right) r_{n-1}^{f} \\
& \times \frac{1}{\prod_{k=n}^{T-1} r_{k}^{f}} r_{n-1}^{e}{ }^{\prime}\left(\sigma_{n-1}\right)^{-1} r_{n-1}^{e} \\
& +\frac{1}{4 \omega^{2}} \frac{1}{\prod_{k=n}^{T-1}\left(r_{k}^{f}\right)^{2}} r_{n-1}^{e}{ }^{\prime}\left(\sigma_{n-1}\right)^{-1} r_{n-1}^{e} \\
& \times\left[1+r_{n-1}^{e}{ }^{\prime}\left(\sigma_{n-1}\right)^{-1} r_{n-1}^{e}\right] .
\end{aligned}
$$

By repeatedly using recursive equation (39), we can obtain (35). Substituting (35) into (40) yields

$$
\begin{aligned}
E\left[\left(X_{n}^{\widehat{\pi}}\right)^{2}\right]= & E\left[\left(X_{n-1}^{\hat{\pi}}\right)^{2}\right]\left(r_{n-1}^{f}\right)^{2} \\
& +\frac{1}{\omega} \frac{r_{n-1}^{e}{ }^{\prime}\left(\sigma_{n-1}\right)^{-1} r_{n-1}^{e}}{\prod_{k=n}^{T-1}\left(r_{k}^{f}\right)^{2}} x_{0} \prod_{k=0}^{T-1} r_{k}^{f} \\
& +\frac{1}{4 \omega^{2}} \frac{r_{n-1}^{e}\left(\sigma_{n-1}\right)^{-1} r_{n-1}^{e}}{\prod_{k=n}^{T-1}\left(r_{k}^{f}\right)^{2}} \\
& \times\left[1+\sum_{k=0}^{n-2} r_{k}^{e \prime}\left(\sigma_{k}\right)^{-1} r_{k}^{e}+\sum_{k=0}^{n-1} r_{k}^{e^{\prime}}\left(\sigma_{k}\right)^{-1} r_{k}^{e}\right] .
\end{aligned}
$$

Repeatedly using the above recursive equation yields (36).

Then according to (35) and (36), we can obtain the efficient frontier under the time-consistent strategy.

Theorem 5. The efficient frontier under the time-consistent strategy (16) is

$$
\operatorname{Var}\left(X_{T}^{\widehat{\pi}}\right)=\frac{\left[E\left(X_{T}^{\widehat{\pi}}\right)-x_{0} \prod_{k=0}^{T-1} r_{k}^{f}\right]^{2}}{\sum_{k=0}^{T-1} r_{k}^{e^{\prime}}\left(\sigma_{k}\right)^{-1} r_{k}^{e}} .
$$

Proof. When $n=T$, (35) becomes

$$
E\left(X_{T}^{\widehat{\pi}}\right)=x_{0} \prod_{k=0}^{T-1} r_{k}^{f}+\frac{1}{2 \omega} \sum_{k=0}^{T-1} r_{k}^{e^{\prime}}\left(\sigma_{k}\right)^{-1} r_{k}^{e}
$$

Equation (43), as we expect, coincides with the expression of $g_{0}(x)$ in (18).

When $n=T$, (36) becomes

$$
\begin{aligned}
& E\left[\left(X_{T}^{\hat{\pi}}\right)^{2}\right] \\
& =\left(x_{0}\right)^{2} \prod_{k=0}^{T-1}\left(r_{k}^{f}\right)^{2}+\frac{1}{\omega} \sum_{k=0}^{T-1} r_{k}^{e^{\prime}}\left(\sigma_{k}\right)^{-1} r_{k}^{e} x_{0} \prod_{k=0}^{T-1} r_{k}^{f} \\
& +\frac{1}{4 \omega^{2}} \sum_{k=0}^{T-1} r_{k}^{e^{\prime}}\left(\sigma_{k}\right)^{-1} r_{k}^{e}\left[1+\sum_{m=0}^{k-1} r_{m}^{e}\left(\sigma_{m}\right)^{-1} r_{m}^{e}\right. \\
& \left.+\sum_{m=0}^{k} r_{m}^{e^{\prime}}\left(\sigma_{m}\right)^{-1} r_{m}^{e}\right]
\end{aligned}
$$




$$
\begin{gathered}
=\left(x_{0} \prod_{k=0}^{T-1} r_{k}^{f}+\frac{1}{2 \omega} \sum_{k=0}^{T-1} r_{k}^{e^{\prime}}\left(\sigma_{k}\right)^{-1} r_{k}^{e}\right)^{2} \\
-\frac{1}{4 \omega^{2}}\left(\sum_{k=0}^{T-1} r_{k}^{e^{\prime}}\left(\sigma_{k}\right)^{-1} r_{k}^{e}\right)^{2} \\
+\frac{1}{4 \omega^{2}} \sum_{k=0}^{T-1} r_{k}^{e^{\prime}}\left(\sigma_{k}\right)^{-1} r_{k}^{e}\left[1+\sum_{m=0}^{k-1} r_{m}^{e^{\prime}}\left(\sigma_{m}\right)^{-1} r_{m}^{e}\right. \\
\left.+\sum_{m=0}^{k} r_{m}^{e^{\prime}}\left(\sigma_{m}\right)^{-1} r_{m}^{e}\right] .
\end{gathered}
$$

Equation (44) together with (43) yields

$$
\begin{aligned}
\operatorname{Var}\left(X_{T}^{\widehat{\pi}}\right) & \frac{1}{4 \omega^{2}} \sum_{k=0}^{T-1} r_{k}^{e^{\prime}}\left(\sigma_{k}\right)^{-1} r_{k}^{e} \\
& \times\left[1+\sum_{m=0}^{k-1} r_{m}^{e^{\prime}}\left(\sigma_{m}\right)^{-1} r_{m}^{e}+\sum_{m=0}^{k} r_{m}^{e^{\prime}}\left(\sigma_{m}\right)^{-1} r_{m}^{e}\right. \\
= & \frac{1}{4 \omega^{2}} \sum_{k=0}^{T-1} r_{k}^{e^{\prime}}\left(\sigma_{k}\right)^{-1} r_{k}^{e} \\
& \left.\times \sum_{k=0}^{T-1} r_{k}^{e^{\prime}}\left(\sigma_{k}\right)^{-1} r_{k}^{e}\right] \\
= & \frac{1}{4 \omega^{2}} \sum_{k=0}^{T-1} r_{k}^{e^{\prime}}\left(\sigma_{k}\right)^{-1} r_{k}^{e} . \\
= & \left.\frac{1}{4 \omega^{2}} \sum_{k=0}^{T-1} r_{k}^{e^{\prime}}\left(\sigma_{k}\right)^{-1} r_{k}^{e} r_{m}^{e}{ }^{e}\left(\sigma_{m}\right)^{-1} r_{m}^{e}-\sum_{m=k}^{T-1} r_{m}^{e}\left(\sigma_{m}\right)^{-1} r_{m}^{e}\right] \\
& \times\left[1+\sum_{m=k}^{T-1} r_{m}^{e}{ }^{e}\left(\sigma_{m}\right)^{-1} r_{m}^{e}-\sum_{m=k}^{T-1} r_{m}^{e}{ }^{\prime}\left(\sigma_{m}\right)^{-1} r_{m}^{e}\right]
\end{aligned}
$$

Referring to (43) and (45), we get the efficient frontier as follows:

$$
\operatorname{Var}\left(X_{T}^{\widehat{\pi}}\right)=\frac{\left[E\left(X_{T}^{\widehat{\pi}}\right)-x_{0} \prod_{k=0}^{T-1} r_{k}^{f}\right]^{2}}{\sum_{k=0}^{T-1} r_{k}^{e^{\prime}}\left(\sigma_{k}\right)^{-1} r_{k}^{e}}
$$

Now we want to compare efficient frontier (42) with the one in [2].
Efficient frontier in [2] is

$$
\begin{aligned}
\operatorname{Var}\left(X_{T}^{\pi^{*}}\right)= & \frac{\prod_{k=0}^{T-1}\left(1-r_{k}^{e \prime} E^{-1}\left(R_{k}^{e} R_{k}^{e \prime}\right) r_{k}^{e}\right)}{1-\prod_{k=0}^{T-1}\left(1-r_{k}^{e \prime} E^{-1}\left(R_{k}^{e} R_{k}^{e \prime}\right) r_{k}^{e}\right)} \\
& \times\left[E\left(X_{T}^{\pi^{*}}\right)-x_{0} \prod_{k=0}^{T-1} r_{k}^{f}\right]^{2} .
\end{aligned}
$$

In view of (32), we can find that

$$
\frac{\prod_{k=0}^{T-1}\left(1-r_{k}^{e \prime} E^{-1}\left(R_{k}^{e} R_{k}^{e \prime}\right) r_{k}^{e}\right)}{1-\prod_{k=0}^{T-1}\left(1-r_{k}^{e \prime} E^{-1}\left(R_{k}^{e} R_{k}^{e \prime}\right) r_{k}^{e}\right)} \leq \frac{1}{\sum_{k=0}^{T-1} r_{k}^{e \prime}\left(\sigma_{k}\right)^{-1} r_{k}^{e}}
$$

Therefore, we have given a mathematical proof to the fact that the efficient frontier for the time-consistent strategy is never above the efficient frontier for the precommitment strategy. Moreover, the shorter the investment horizon $T$ is, the closer these two efficient frontiers are.

3.3. Numerical Analysis. In this part, we want to compare expected terminal wealth and efficient frontier under timeconsistent framework with the corresponding ones, respectively, in [2]. Let initial wealth $x_{0}=1$. For convenience, we assume that there are only one risk-free asset and one risky asset in the market. Furthermore, we suppose that the riskfree return is a constant $r^{f}=1.15$ during all the investment periods and risky returns $\left\{R_{n}, n=0,1, \ldots, T-1\right\}$ have the same distribution function with the same expectation $E\left(R_{n}\right)=1.35$ and variance $\operatorname{Var}\left(R_{n}\right)=0.2$.

(1) Let risk aversion $\omega=1$. Here we fix other parameters and make $T$ increase from 1 to 8 , to present the effect of investment horizon on the distance between time-consistent expected terminal wealth and precommitment one. Refering to (43) and formula (56) in [2], we obtain Figure 1, which shows that precommitment expected terminal wealth is higher than the time-consistent one and the gap between these two expectations is widened when $T$ increases. Actually, the gap sequences are 0,0.0506, 0.1747, 0.4051, 0.7899, $1.3985,2.3316$, and 3.7352 when $T$ is changed from 1 to 8 . This phenomenon shows that for short time horizons, time inconsistency has a slight effect on the relative results. But for long time horizon, noncooperation of each decision maker destroys the welfare of the whole game, and the longer $T$ is, the larger the loss is.

(2) Let $T=8$. Here we fix other parameters and make risk aversion $\omega$ increase from 1 to 8 . The effect of risk aversion on the distance between time-consistent expected terminal wealth and precommitment one is showed in Figure 2, which indicates that the distance between these two kinds of expected final wealth is minor when risk aversion is big enough. This is because, by (43) and formula (56) in [2], we can find that distance between these two expectations are only affected by the risky investment income. When $\omega$ is big enough, either the time-consistent investor or the precommitment one will prefer to invest less wealth in risky asset, which leads to the conclusion in Figure 2. 


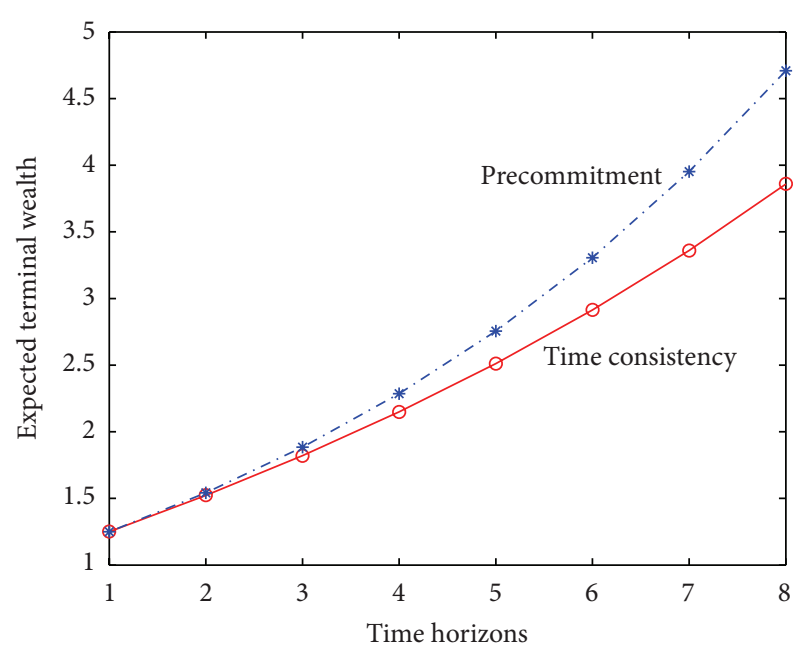

FIGURE 1: Effect of time horizons.

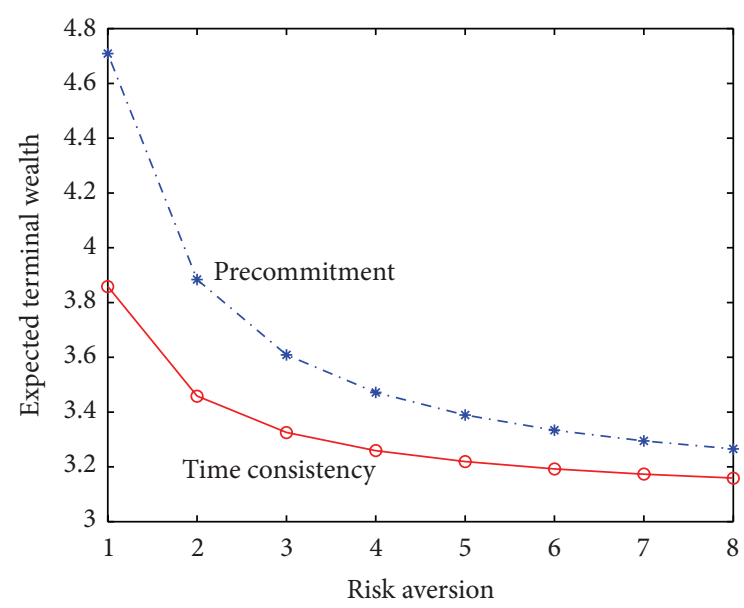

FIGURE 2: Effect of the risk aversion.

(3) We want to compare the precommitment efficient frontier with the time-consistent one when $T=4,8$ and $\omega=$ 2. Conclusions showed in Figure 3 coincide with our conclusions derived by mathematical analysis in Section 3.2.3 and the reason behind Figure 3 is similar to that demonstrated in Figure 1.

\section{Nash Equilibrium Strategy with Wealth-Dependent Risk Aversion}

In this section, we will consider an optimization problem

$$
\max _{\pi(n)}\left\{E_{n, x}\left(X_{T}^{\pi(n)}\right)-\omega(x) \operatorname{Var}_{n, x}\left(X_{T}^{\pi(n)}\right)\right\}
$$

under the framework of Nash equilibrium, where the risk aversion $\omega(x)$ is a function of current wealth $x$. We just study a tractable situation that the risk aversion is inversely proportional to wealth according to some psychological results; that is, $\omega(x)=\omega / x$ where constant $\omega>0$ is called the coefficient of risk aversion. It still remains open what the time-consistent strategy is with other forms of $\omega(x)$.

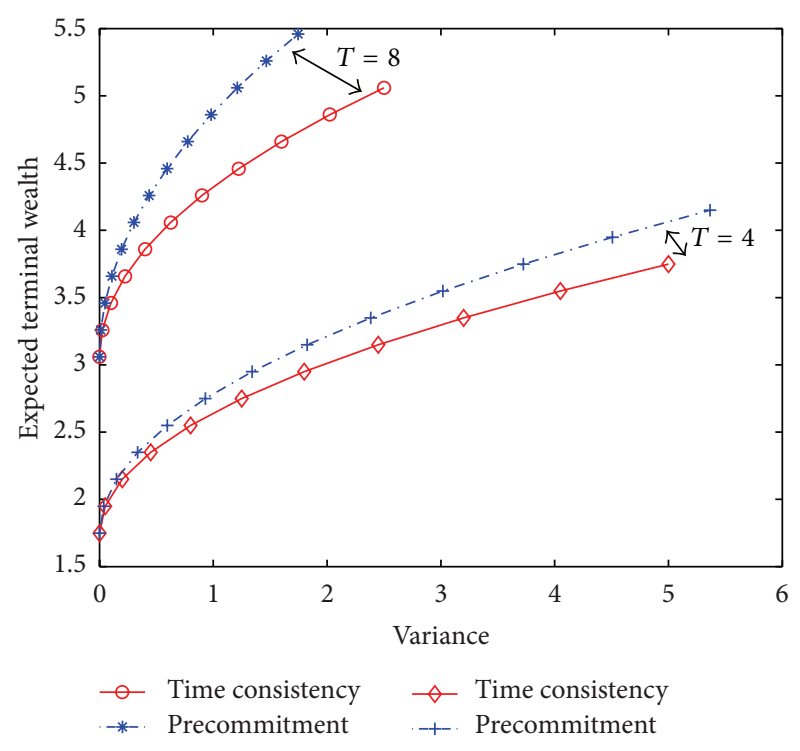

FIGURE 3: Efficient frontier.

4.1. Nash Equilibrium Strategy and Equilibrium Value Function. Similarly, referring to (8), the recursion of $V_{n}(x)$ is

$$
\begin{aligned}
V_{n}(x)=\max _{\pi_{n}}\left\{E_{n, x}\left[V_{n+1}\left(X_{n+1}^{\pi_{n}}\right)\right]\right. \\
+E_{n, x}\left[\omega\left(X_{n+1}^{\pi_{n}}\right) h_{n+1}\left(X_{n+1}^{\pi_{n}}\right)\right. \\
\left.-\omega(x) h_{n+1}\left(X_{n+1}^{\pi_{n}}\right)\right] \\
-E_{n, x}\left[\omega\left(X_{n+1}^{\pi_{n}}\right)\left(g_{n+1}\left(X_{n+1}^{\pi_{n}}\right)\right)^{2}\right] \\
\left.+\omega(x)\left[E_{n, x}\left(g_{n+1}\left(X_{n+1}^{\pi_{n}}\right)\right)\right]^{2}\right\}, \\
n=0,1, \ldots, T-1,
\end{aligned}
$$

with terminal condition $V_{T}(x)=x$.

The recursions of $g_{n}(x)$ and $h_{n}(x)$ are, respectively,

$$
\begin{aligned}
& g_{n}(x)=E\left[X_{T}^{\widehat{\pi}(n)} \mid X_{n}=x\right] \\
&=E_{n, x}\left(g_{n+1}\left(X_{n+1}^{\widehat{\pi}_{n}}\right)\right), \quad n=0,1, \ldots, T-1, \\
& g_{T}(x)=x, \\
& h_{n}(x)=E\left[\left(X_{T}^{\hat{\pi}(n)}\right)^{2} \mid X_{n}=x\right] \\
&=E_{n, x}\left(h_{n+1}\left(X_{n+1}^{\widehat{\pi}_{n}}\right)\right), \quad n=0,1, \ldots, T-1, \\
& h_{T}(x)=x^{2} .
\end{aligned}
$$

Before giving the time-consistent results, we need to introduce the following sequences $\left\{a_{n}\right\}$ and $\left\{b_{n}\right\}$ and their properties:

$$
\begin{aligned}
& a_{T}=1, \\
& b_{T}=1,
\end{aligned}
$$




$$
\begin{gathered}
\xi_{n+1}=b_{n+1} E\left(R_{n}^{e} R_{n}^{e \prime}\right)-\left(a_{n+1}\right)^{2} r_{n}^{e} r_{n}^{e \prime}, \\
n=T-1, T-2, \ldots, 0, \\
a_{n}=a_{n+1}\left(r_{n}^{f}+\frac{a_{n+1}+2 \omega\left(\left(a_{n+1}\right)^{2}-b_{n+1}\right) r_{n}^{f}}{2 \omega}\right. \\
\left.\quad \times r_{n}^{e^{\prime}}\left(\xi_{n+1}\right)^{-1} r_{n}^{e}\right), \quad n=T-1, T-2, \ldots, 0, \\
b_{n}=b_{n+1} E \quad\left(r_{n}^{f}+\frac{a_{n+1}+2 \omega\left(\left(a_{n+1}\right)^{2}-b_{n+1}\right) r_{n}^{f}}{2 \omega}\right. \\
\left.\quad \times r_{n}^{e \prime}\left(\xi_{n+1}\right)^{-1} R_{n}^{e}\right), \quad n=T-1, T-2, \ldots, 0 .
\end{gathered}
$$

Lemma 6. For $n=0,1, \ldots, T-1, b_{n}>\left(a_{n}\right)^{2}$ and $\xi_{n}(n=$ $T, T-1, \ldots, 1)$ is positive definite.

Proof. $\xi_{T}=E\left(R_{T-1}^{e} R_{T-1}^{e}{ }^{\prime}\right)-r_{T-1}^{e} r_{T-1}^{e}{ }^{\prime}=\sigma_{T-1}$ is obviously positive definite. Then,

$$
\begin{aligned}
b_{T-1}=E & {\left[\left(r_{T-1}^{f}+\frac{1}{2 \omega} r_{T-1}^{e}{ }^{\prime}\right.\right.} \\
& \left.\left.\times\left(E\left(R_{T-1}^{e} R_{T-1}^{e}{ }^{\prime}\right)-r_{T-1}^{e} r_{T-1}^{e}{ }^{\prime}\right)^{-1} R_{T-1}^{e}\right)^{2}\right], \\
a_{T-1}= & r_{T-1}^{f}+\frac{1}{2 \omega} r_{T-1}^{e}{ }^{\prime} \\
& \times\left(E\left(R_{T-1}^{e} R_{T-1}^{e}{ }^{\prime}-r_{T-1}^{e} r_{T-1}^{e}{ }^{\prime}\right)\right)^{-1} r_{T-1}^{e},
\end{aligned}
$$

and hence $b_{T-1}>\left(a_{T-1}\right)^{2}$. Now for any column vector $r \neq \mathbf{0}$, we have

$$
\begin{aligned}
r^{\prime} \xi_{T-1} r & =r^{\prime}\left(b_{T-1} E\left(R_{T-2}^{e} R_{T-2}^{e}{ }^{\prime}\right)-\left(a_{T-1}\right)^{2} r_{T-2}^{e} r_{T-2}^{e}{ }^{\prime}\right) r \\
& >r^{\prime}\left(\left(a_{T-1}\right)^{2} E\left(R_{T-2}^{e} R_{T-2}^{e}{ }^{\prime}\right)-\left(a_{T-1}\right)^{2} r_{T-2}^{e} r_{T-2}^{e}{ }^{\prime}\right) r \\
& =\left(a_{T-1}\right)^{2} r^{\prime} \sigma_{T-2} r \\
& >0
\end{aligned}
$$

and then $\xi_{T-1}$ is positive definite.
Now we assume that for $n+1, b_{n+1}>\left(a_{n+1}\right)^{2}$ and $\xi_{n+1}$ is positive definite, and then for $n$,

$$
\begin{aligned}
& b_{n}-\left(a_{n}\right)^{2} \\
& =b_{n+1} E\left[\left(r_{n}^{f}+\frac{a_{n+1}+2 \omega\left(\left(a_{n+1}\right)^{2}-b_{n+1}\right) r_{n}^{f}}{2 \omega}\right.\right. \\
& \left.\left.\times r_{n}^{e^{\prime}}\left(\xi_{n+1}\right)^{-1} R_{n}^{e}\right)^{2}\right] \\
& -\left(a_{n+1}\right)^{2}\left(r_{n}^{f}+\frac{a_{n+1}+2 \omega\left(\left(a_{n+1}\right)^{2}-b_{n+1}\right) r_{n}^{f}}{2 \omega}\right. \\
& \left.\times r_{n}^{e^{\prime}}\left(\xi_{n+1}\right)^{-1} r_{n}^{e}\right)^{2} \\
& >\left(a_{n+1}\right)^{2} E\left[\left(r_{n}^{f}+\frac{a_{n+1}+2 \omega\left(\left(a_{n+1}\right)^{2}-b_{n+1}\right) r_{n}^{f}}{2 \omega}\right.\right. \\
& \left.\left.\times r_{n}^{e^{\prime}}\left(\xi_{n+1}\right)^{-1} R_{n}^{e}\right)^{2}\right] \\
& -\left(a_{n+1}\right)^{2}\left(r_{n}^{f}+\frac{a_{n+1}+2 \omega\left(\left(a_{n+1}\right)^{2}-b_{n+1}\right) r_{n}^{f}}{2 \omega}\right. \\
& \left.\times r_{n}^{e^{\prime}}\left(\xi_{n+1}\right)^{-1} r_{n}^{e}\right)^{2}>0 .
\end{aligned}
$$

Similar to the proof of $\xi_{T-1}$ which is positive definite, we can immediately prove that $\xi_{n}$ is positive definite given $b_{n}>\left(a_{n}\right)^{2}$. By induction, we complete the proof of Lemma 6.

The recursions (50)-(51) and Lemma 6 yield the following theorem.

Theorem 7. If $x>0$, the time-consistent strategy is

$$
\begin{array}{r}
\hat{\pi}_{n}(x)=\frac{a_{n+1}+2 \omega\left(\left(a_{n+1}\right)^{2}-b_{n+1}\right) r_{n}^{f}}{2 \omega}\left(\xi_{n+1}\right)^{-1} r_{n}^{e} x, \\
n=0,1, \ldots, T-1,
\end{array}
$$

and the equilibrium strategy is

$$
\begin{aligned}
V_{n}(x)= & a_{n} x+\omega\left(a_{n}\right)^{2} x-\omega b_{n} x, \quad n=0,1, \ldots, T, \\
& g_{n}(x)=a_{n} x, \quad n=0,1, \ldots, T-1, \\
& h_{n}(x)=b_{n} x^{2}, \quad n=0,1, \ldots, T-1 .
\end{aligned}
$$

If $x \leq 0, V_{n}(x)=g_{n}(x)=h_{n}(x)=+\infty$ and the model is meaningless. 
Remark 8. If the initial wealth is big enough and risky assets have steady returns, then at each period wealth level is often greater than 0 in the real-world situation. Therefore, the condition $x>0$ is not a severe requirement.

Proof. By (50), we obtain

$$
\begin{aligned}
& V_{T-1}(x)=\max _{\pi_{T-1}}\left\{E_{T-1, x}\left[V_{T}\left(X_{T}^{\pi_{T-1}}\right)\right]\right. \\
& +E_{T-1, x}\left[\omega\left(X_{T}^{\pi_{T-1}}\right) h_{T}\left(X_{T}^{\pi_{T-1}}\right)\right. \\
& \left.-\omega(x) h_{T}\left(X_{T}^{\pi_{T-1}}\right)\right] \\
& -E_{T-1, x}\left[\omega\left(X_{T}^{\pi_{T-1}}\right)\left(g_{T}\left(X_{T}^{\pi_{T-1}}\right)\right)^{2}\right] \\
& \left.+\omega(x)\left[E_{T-1, x}\left(g_{T}\left(X_{T}^{\pi_{T-1}}\right)\right)\right]^{2}\right\} \\
& =\max _{\pi_{T-1}}\left\{E_{T-1, x}\left(X_{T}^{\pi_{T-1}}\right)\right. \\
& \left.-\omega(x) \operatorname{Var}_{T-1, x}\left(X_{T}^{\pi_{T-1}}\right)\right\} \\
& =\max _{\pi_{T-1}}\left\{x r_{T-1}^{f}+\pi_{T-1}^{\prime} r_{T-1}^{e}\right. \\
& \left.-\omega(x) \pi_{T-1}^{\prime} \sigma_{T-1} \pi_{T-1}\right\} .
\end{aligned}
$$

It is obvious that when $x>0$,

$$
\widehat{\pi}_{T-1}(x)=\frac{1}{2 \omega(x)}\left(\sigma_{T-1}\right)^{-1} r_{T-1}^{e}=\frac{1}{2 \omega}\left(\sigma_{T-1}\right)^{-1} r_{T-1}^{e} x .
$$

Then,

$$
\begin{aligned}
g_{T-1}(x) & =E_{T-1, x}\left(X_{T}^{\hat{\pi}_{T-1}}\right) \\
& =x r_{T-1}^{f}+\frac{1}{2 \omega(x)} r_{T-1}^{e}{ }^{\prime}\left(\sigma_{T-1}\right)^{-1} r_{T-1}^{e} \\
& =\left(r_{T-1}^{f}+\frac{\kappa_{T-1}}{2 \omega}\right) x=a_{T-1} x, \\
h_{T-1}(x) & =E_{T-1, x}\left[\left(X_{T}^{\widehat{\pi}_{T-1}}\right)^{2}\right] \\
& =E_{T-1, x}\left[\left(x r_{T-1}^{f}+\widehat{\pi}_{T-1}^{\prime} R_{T-1}^{e}\right)^{2}\right] \\
& =x^{2}\left(r_{T-1}^{f}\right)^{2}+\frac{r_{T-1}^{f} \kappa_{T-1}}{\omega(x)} x+\frac{\kappa_{T-1}\left(1+\kappa_{T-1}\right)}{4(\omega(x))^{2}} \\
& =\left(\left(r_{T-1}^{f}\right)^{2}+\frac{r_{T-1}^{f} \kappa_{T-1}}{\omega}+\frac{\kappa_{T-1}\left(1+\kappa_{T-1}\right)}{4 \omega^{2}}\right) x^{2} \\
& =b_{T-1} x^{2} .
\end{aligned}
$$

Substituting (61) into $V_{T-1}(x)$ yields

$$
\begin{aligned}
V_{T-1}(x) & =x r_{T-1}^{f}+\frac{1}{4 \omega(x)} r_{T-1}^{e}{ }^{\prime}\left(\sigma_{T-1}\right)^{-1} r_{T-1}^{e} \\
& =\left(r_{T-1}^{f}+\frac{1}{4 \omega} \kappa_{T-1}\right) x \\
& =\left(a_{T-1}+\omega\left(a_{T-1}\right)^{2}-\omega b_{T-1}\right) x .
\end{aligned}
$$

Equations (61)-(64) mean that (56)-(59) are true for $n=T-$ 1.

We assume the results in Theorem 7 hold true for $n+1$, and then according to (50) and (57),

$$
\begin{aligned}
& V_{n}(x)=\max _{\pi_{n}}\left\{E_{n, x}\left[\left(a_{n+1}+\omega\left(a_{n+1}\right)^{2}-\omega b_{n+1}\right) X_{n+1}^{\pi_{n}}\right]\right. \\
& +E_{n, x}\left[\omega b_{n+1} X_{n+1}^{\pi_{n}}-\frac{\omega}{x} b_{n+1}\left(X_{n+1}^{\pi_{n}}\right)^{2}\right] \\
& -E_{n, x}\left[\omega\left(a_{n+1}\right)^{2} X_{n+1}^{\pi_{n}}\right] \\
& \left.+\frac{\omega}{x}\left[E_{n, x}\left(a_{n+1} X_{n+1}^{\pi_{n}}\right)\right]^{2}\right\} \\
& =\max _{\pi_{n}}\left\{E_{n, x}\left[a_{n+1} X_{n+1}^{\pi_{n}}-\frac{\omega}{x} b_{n+1}\left(X_{n+1}^{\pi_{n}}\right)^{2}\right]\right. \\
& \left.+\frac{\omega}{x}\left[E_{n, x}\left(a_{n+1} X_{n+1}^{\pi_{n}}\right)\right]^{2}\right\} \\
& =\max _{\pi_{n}}\left\{E _ { n , x } \left[a_{n+1}\left(x r_{n}^{f}+\pi_{n}^{\prime} R_{n}^{e}\right)\right.\right. \\
& \left.-\frac{\omega}{x} b_{n+1}\left(x r_{n}^{f}+\pi_{n^{\prime}} R_{n}^{e}\right)^{2}\right] \\
& \left.+\frac{\omega}{x}\left[a_{n+1}\left(x r_{n}^{f}+\pi_{n}^{\prime} r_{n}^{e}\right)\right]^{2}\right\} . \\
& =\max _{\pi_{n}}\left\{a_{n+1}\left(x r_{n}^{f}+\pi_{n}^{\prime} r_{n}^{e}\right)\right. \\
& -\frac{\omega}{x} b_{n+1}\left(x^{2}\left(r_{n}^{f}\right)^{2}+2 x r_{n}^{f} \pi_{n}^{\prime} r_{n}^{e}\right. \\
& \left.+\pi_{n}^{\prime} E\left(R_{n}^{e} R_{n}^{e^{\prime}}\right) \pi_{n}\right) \\
& \left.+\frac{\omega}{x}\left[a_{n+1}\left(x r_{n}^{f}+\pi_{n}^{\prime} r_{n}^{e}\right)\right]^{2}\right\} .
\end{aligned}
$$

By Lemma 6, we know that $\xi_{n+1}=b_{n+1} E\left(R_{n}^{e} R_{n}^{e^{\prime}}\right)-\left(a_{n+1}\right)^{2} r_{n}^{e} r_{n}^{e^{\prime}}$ is positive definite. Given that $x>0$, we can see that the optimal solution of (66) exists and is

$$
\begin{aligned}
\hat{\pi}_{n}(x)= & \frac{a_{n+1}+2 \omega\left(\left(a_{n+1}\right)^{2}-b_{n+1}\right) r_{n}^{f}}{2 \omega} \\
& \times\left(b_{n+1} E\left(R_{n}^{e} R_{n}^{e^{\prime}}\right)-\left(a_{n+1}\right)^{2} r_{n}^{e} r_{n}^{e^{\prime}}\right)^{-1} r_{n}^{e} x .
\end{aligned}
$$


Then,

$$
\begin{aligned}
g_{n}(x)= & E_{n, x}\left(g_{n+1}\left(X_{n+1}^{\widehat{\pi}_{n}}\right)\right) \\
= & E_{n, x}\left(a_{n+1}\left(x r_{n}^{f}+\hat{\pi}_{n}^{\prime} R_{n}^{e}\right)\right) \\
= & a_{n+1}\left(r_{n}^{f}+\frac{a_{n+1}+2 \omega\left(\left(a_{n+1}\right)^{2}-b_{n+1}\right) r_{n}^{f}}{2 \omega}\right. \\
& \left.\times r_{n}^{e \prime}\left(\xi_{n+1}\right)^{-1} r_{n}^{e}\right) x \equiv a_{n} x, \\
h_{n}(x)= & E_{n, x}\left[b_{n+1}\left(X_{n+1}^{\hat{\pi}_{n}}\right)^{2}\right] \\
= & b_{n+1} E_{n, x}\left[\left(x r_{n}^{f}+\hat{\pi}_{n}^{\prime} R_{n}^{e}\right)^{2}\right] \\
= & {\left[\left(r _ { n + 1 } ^ { f } E \left[\frac{a_{n+1}+2 \omega\left(\left(a_{n+1}\right)^{2}-b_{n+1}\right) r_{n}^{f}}{2 \omega}\right.\right.\right.} \\
& \left.\left.\quad \times r_{n}^{e \prime}\left(\xi_{n+1}\right)^{-1} R_{n}^{e}\right)^{2}\right] x^{2} \equiv b_{n} x^{2} .
\end{aligned}
$$

According to (65), we have

$$
\begin{aligned}
& V_{n}(x)=a_{n+1}\left(r_{n}^{f}+\frac{a_{n+1}+2 \omega\left(\left(a_{n+1}\right)^{2}-b_{n+1}\right) r_{n}^{f}}{2 \omega}\right. \\
& \left.\times r_{n}^{e^{\prime}}\left(\xi_{n+1}\right)^{-1} r_{n}^{e}\right) x \\
& -\omega b_{n+1} E\left(r_{n}^{f}+\frac{a_{n+1}+2 \omega\left(\left(a_{n+1}\right)^{2}-b_{n+1}\right) r_{n}^{f}}{2 \omega}\right. \\
& \left.\times r_{n}^{e \prime}\left(\xi_{n+1}\right)^{-1} R_{n}^{e}\right)^{2} x \\
& +\omega\left(a_{n+1}\right)^{2}\left(r_{n}^{f}+\frac{a_{n+1}+2 \omega\left(\left(a_{n+1}\right)^{2}-b_{n+1}\right) r_{n}^{f}}{2 \omega}\right. \\
& \left.\times r_{n}^{e \prime}\left(\xi_{n+1}\right)^{-1} r_{n}^{e}\right)^{2} x \\
& =a_{n} x+\omega\left(a_{n}\right)^{2} x-\omega b_{n} x .
\end{aligned}
$$

Therefore, we complete the proof of Theorem 7 .

4.2. Numerical Analysis. In this part, for convenience, we assume that there are only one risk-free asset and one risky asset in the market. Furthermore, we suppose that the riskfree return is a constant $r^{f}=1.15$ during all the investment

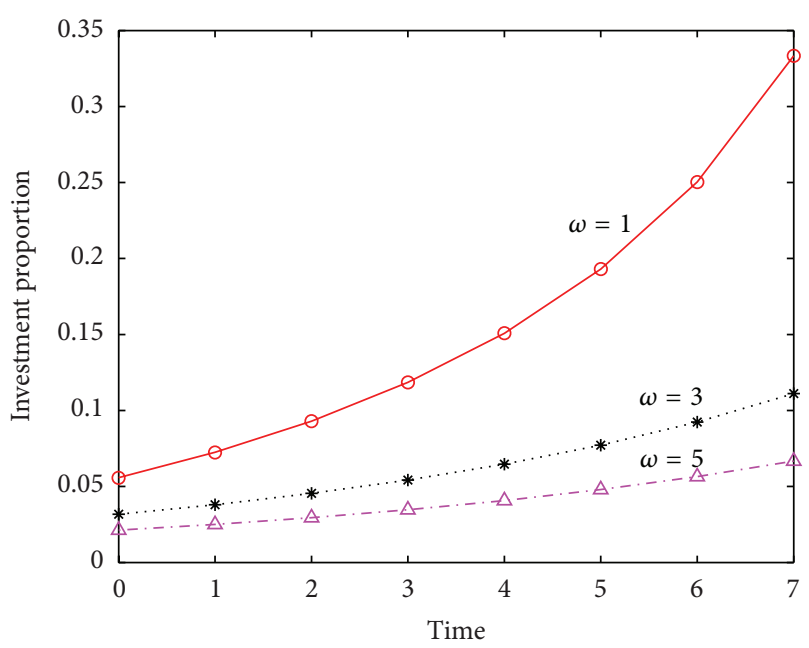

FIGURE 4: Effect of coefficient of risk aversion on the investment proportion.

periods and risky returns $\left\{R_{n}, n=0,1, \ldots, T-1\right\}$ have the same distribution function with the same expectation $E\left(R_{n}\right)=1.35$ and variance $\operatorname{Var}\left(R_{n}\right)=0.3$.

Based on the assumption above, time-consistent strategy (56) can be simplified as

$$
\begin{aligned}
\hat{\pi}_{n}= & \frac{a_{n+1}+2 \omega\left(\left(a_{n+1}\right)^{2}-b_{n+1}\right) r^{f}}{b_{n+1}-\left(\left(a_{n+1}\right)^{2}-b_{n+1}\right)\left(\left(r^{e}\right)^{2} / \operatorname{Var}\left(R^{e}\right)\right)} \\
& \times \frac{1}{2 \omega} \frac{r^{e}}{\operatorname{Var}\left(R^{e}\right)} x \quad n=0,1, \ldots, T-1,
\end{aligned}
$$

and we define

$$
\rho_{n}=\frac{a_{n+1}+2 \omega\left(\left(a_{n+1}\right)^{2} b_{n+1}\right) r^{f}}{b_{n+1}-\left(\left(a_{n+1}\right)^{2}-b_{n+1}\right)\left(\left(r^{e}\right)^{2} / \operatorname{Var}\left(R^{e}\right)\right)} \frac{1}{2 \omega} \frac{r^{e}}{\operatorname{Var}\left(R^{e}\right)}
$$

as proportion invested in the risky asset at time $n$.

(1) First, we want to find the relationship between $\rho_{n}$ and the coefficient of risk aversion $\omega$. Let $T=8$ and coefficient of risk aversion $\omega=1,3,5$, respectively, and then we get Figure 4. From Figure 4, we can find that the smaller the $\omega$, the larger the investment proportion. This is because coefficient $\omega$ now is an index directly proportional to risk aversion, and the higher the $\omega$ is, the more risk aversion the investor has.

(2) Let $\omega$ be a constant 3 and let $T$ take values $4,6,8$, and 10 , respectively, and then we obtain Figure 5, which indicates some interesting phenomena as follows.

(a) In the last 4 periods, no matter whether $T$ is $4,6,8$, or 10 , the investment proportions at each corresponding period take the same values. For an instance, proportions in the final period are the same no matter which value $T$ takes. This is one important character of the time-consistent strategy since the decision at time $n$ under time-consistent framework just depends on the decisions of the forthcoming decision makers. 


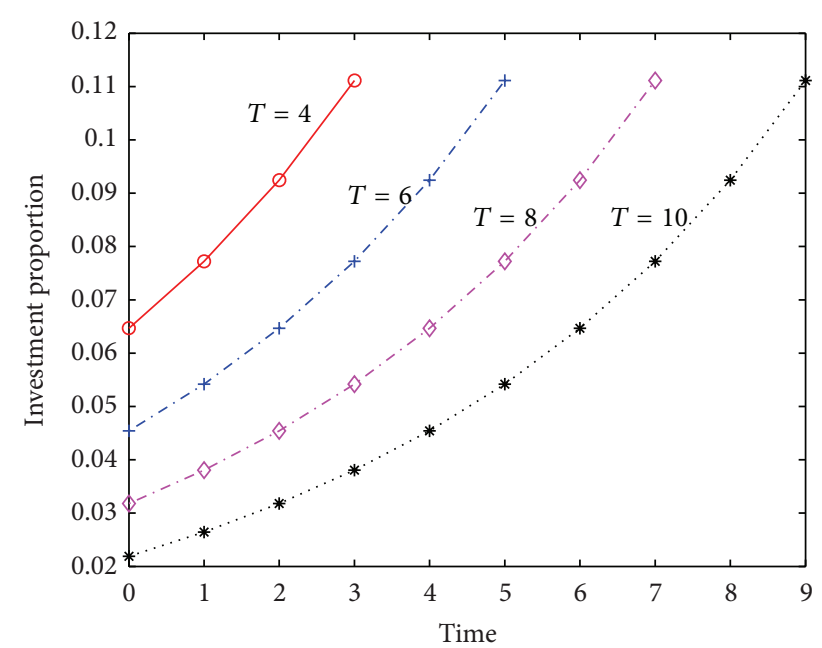

FIGURE 5: Investment proportions with different time horizons.

(b) In the first 4 periods, if investment time horizon $T$ is shorter, $\rho_{n}(n=0,1,2,3)$ is higher. For an instance, when $T=4, \rho_{0}$ is higher than the $\rho_{0}$ when $T=6,8$, and 10 . The reason is that, with shorter investment horizon, the investor will have more confidence to control the uncertainty in the future, which results in a higher investment proportion of the risky asset. In contrast, there is more uncertainty when investment time horizon $T$ is longer, then the investor would rather choose a relatively safer investment strategy, that is, choose a strategy with lower proportion invested in the risky asset.

\section{Conclusions}

It remains prevalent to obtain the pre-commitment strategy for multiperiod mean-variance portfolio selection problems, but not much is known about their time-consistent strategy. This paper aims to investigate the time-consistent Nash equilibrium strategy for a multiperiod mean-variance model. We view this decision-making process as a noncooperative game and suppose that there is one decision maker for each point of time. Two cases are considered in our paper. In the first case, we assume that the risk aversion is a constant and compare our time-consistent results with the precommitment ones. Some desired conclusions are obtained by rigorous proofs. In the second case, the risk aversion depends dynamically on the current wealth. The numerical analysis indicates that time-consistent decision at current time just depends on the decisions of the forthcoming decision makers, which is one important character of time-consistent strategy.

\section{Acknowledgments}

This research is supported by Grants of the MOE Project of the Key Research Institute of Humanities and Social Sciences at Universities of China (no. 11JJD790004), Humanity and Social Science Foundation of Ministry of Education of China (no. 12YJCZH219), and the National Natural Science Foundation of China (no. 71271223, no. 71201173).

\section{References}

[1] H. Markowitz, "Portfolio selection," Journal of Finance, vol. 7, pp. 77-91, 1952.

[2] D. Li and W.-L. Ng, "Optimal dynamic portfolio selection: multiperiod mean-variance formulation," Mathematical Finance, vol. 10, no. 3, pp. 387-406, 2000.

[3] X. Y. Zhou and D. Li, "Continuous-time mean-variance portfolio selection: a stochastic LQ framework," Applied Mathematics and Optimization, vol. 42, no. 1, pp. 19-33, 2000.

[4] S. Basak and G. Chabakauri, "Dynamic mean-variance asset allocation," Review of Financial Studies, vol. 23, pp. 2970-3016, 2010.

[5] R. Strotz, "Myopia and inconsistency in dynamic utility maximization," Review of Economic Studies, vol. 23, pp. 165-180, 1956.

[6] R. Pollak, "Consistent planning," Review of Economic Studies, vol. 35, pp. 185-199, 1968.

[7] B. Peleg and M. E. Yarri, "On the existence of a consistent course of action when tastes are changing," Review of Economic Studies, vol. 40, pp. 391-401, 1973.

[8] I. Ekeland and A. Lazrak, Being Serious about NonCommitment: Subgame Perfect Equilibrium in Continuous Time, Working paper, University of British Columbia, 2006.

[9] I. Ekeland and A. Lazrak, Investment and Consumption without Commitment, Working paper, University of British Columbia, 2008.

[10] T. Björk and A. Murgoci, A General Theory of Markovian time Inconsistent Stochastic Control Problems, Working paper, Stockholm School of Economics, 2010.

[11] T. Björk, A. Murgoci, and X. Y. Zhou, "Mean variance portfolio optimization with state dependent risk aversion," Mathematical Finance, 2012.

[12] E. M. Kryger and M. Steffensen, "Some solvable portfolio problems with quadratic and collective objectives," Working paper, 2010, http://papers.ssrn.com/sol3/papers.cfm? abstract id $=1577265$.

[13] Y. Zeng and Z. F. Li, "Optimal time-consistent investment and reinsurance policies for mean-variance insurers," Insurance: Mathematics \& Economics, vol. 49, no. 1, pp. 145-154, 2011.

[14] J. Wang and P. A. Forsyth, "Continuous time mean variance asset allocation: a time-consistent strategy," European Journal of Operational Research, vol. 209, no. 2, pp. 184-201, 2011.

[15] H. Wang, Time-Inconsistency: Performance of the Local MeanVariance Optimal Portfolio, Working paper, Mathematical Institute, University of Oxford, 2009. 


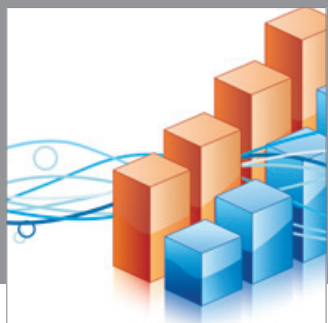

Advances in

Operations Research

mansans

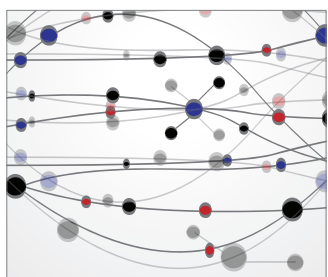

The Scientific World Journal
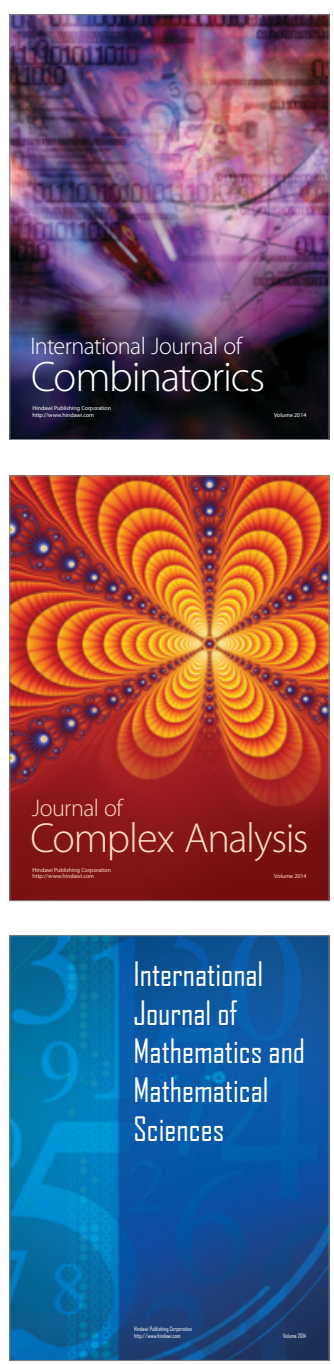
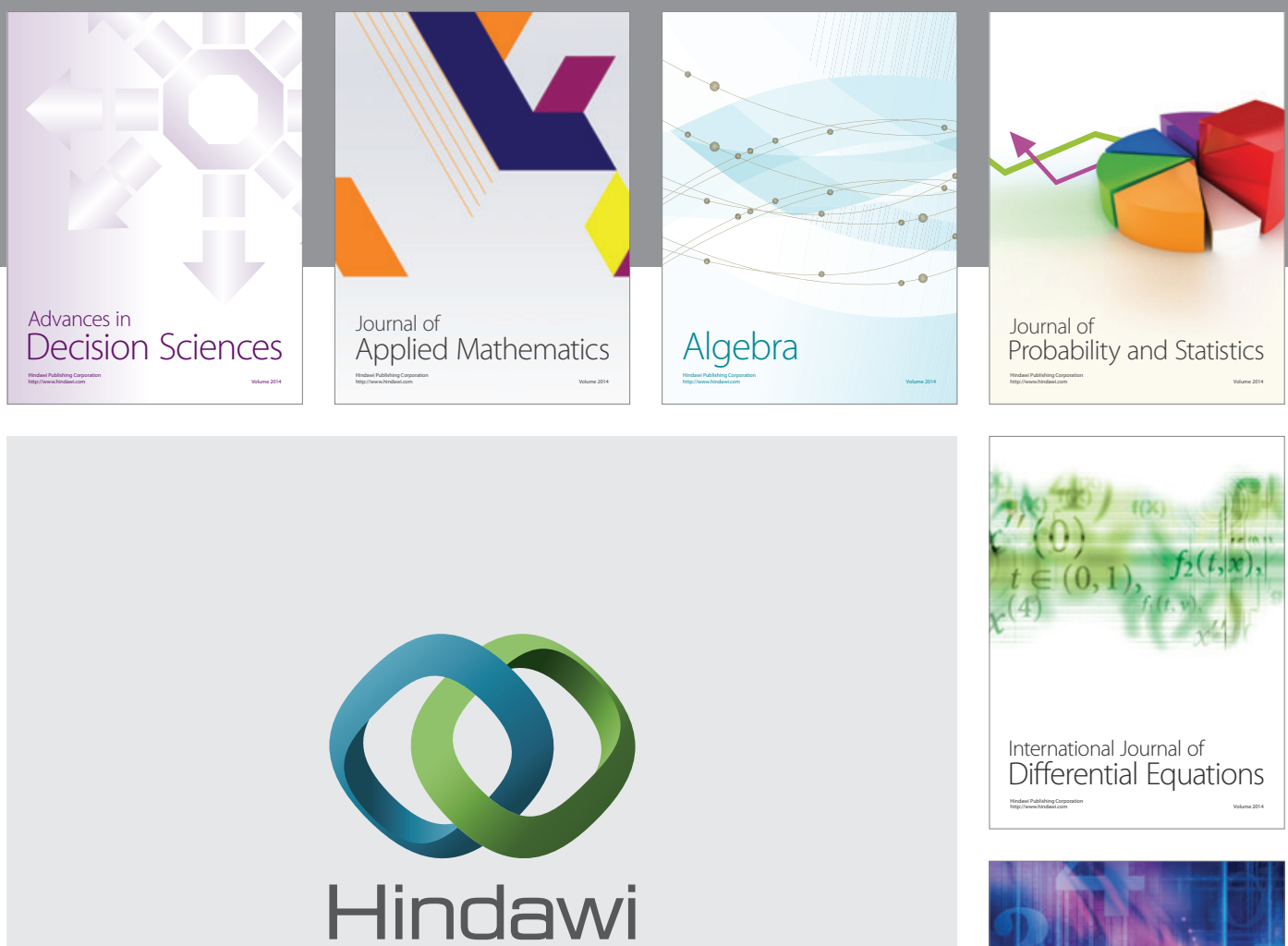

Submit your manuscripts at http://www.hindawi.com
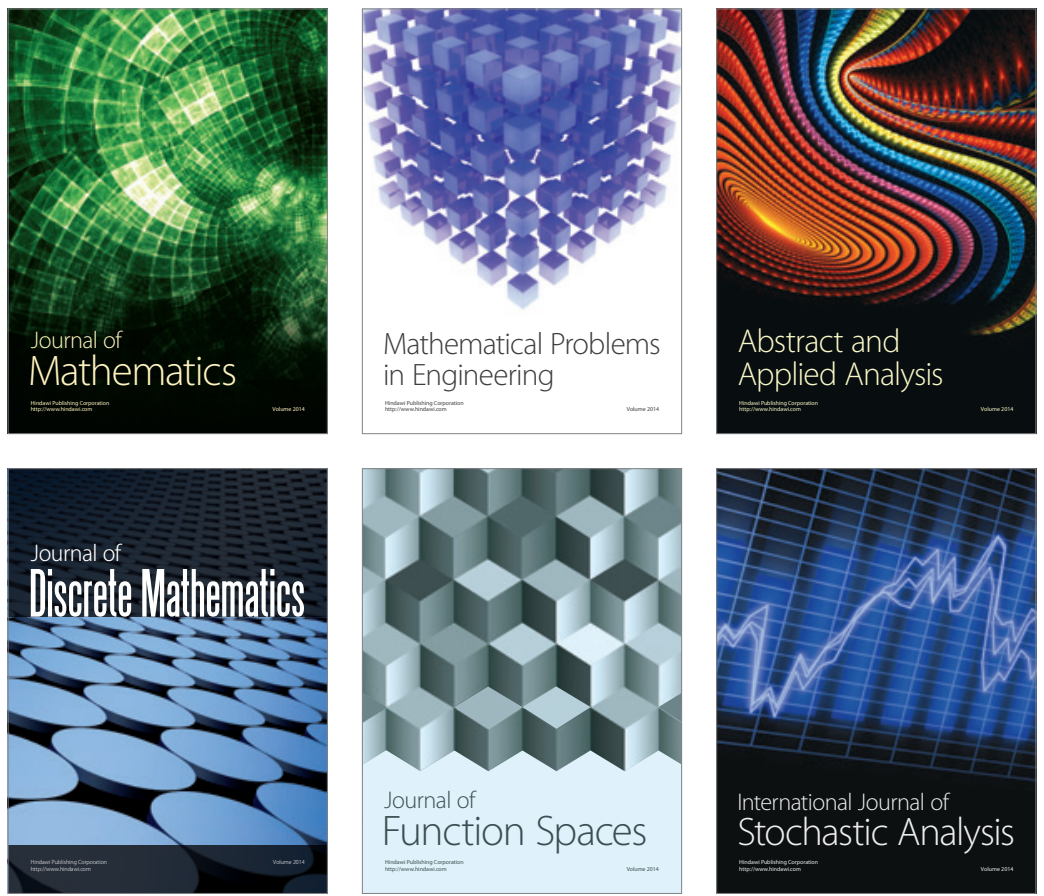

Journal of

Function Spaces

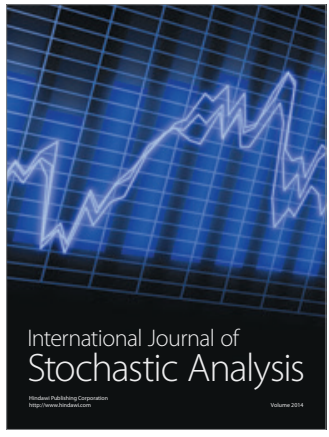

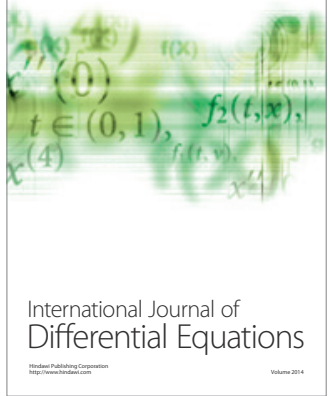
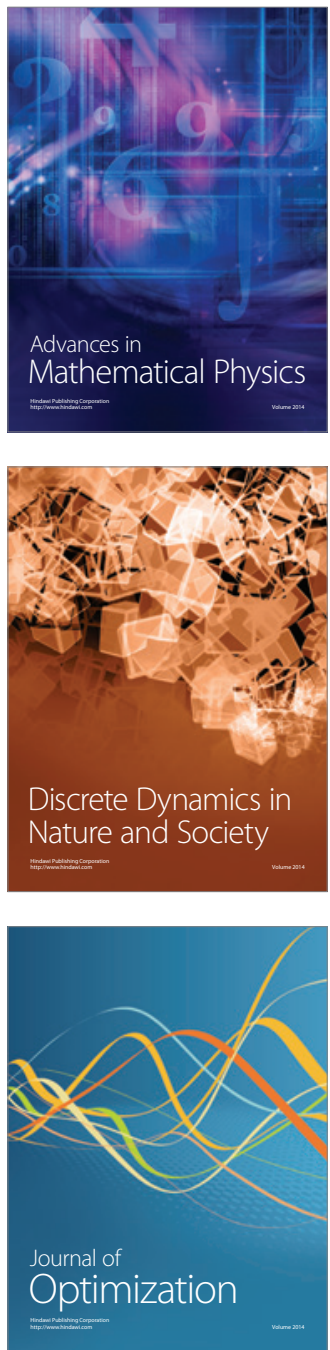